\title{
CES
}

COOPERATIVISMO E ECONOMÍA SOCIAL

Núm. 41 (2018-2019), páxs. 273-282

ISSN: $1130-2682$

\section{CASTILLA Y LEÓN MODIFICA SU LEY DE COOPERATIVAS DE 2002}

\section{CASTILLA Y LEÓN MODIFIES ITS LAW OF COOPERATIVES OF 2002}

\author{
José Antonio Rodríguez MigueZ*
}

\footnotetext{
* Doctor en Derecho. Miembro del IDIUS y CERGI, de la USC. Dirección de correo electrónico:
} jarmiguez@gmail.com 


\title{
RESUMEN
}

La Ley 4/2002, de 11 de abril, de Cooperativas de la Comunidad de Castilla y León es reformada a través de la Ley $2 / 2018$, de 18 de junio, dirigida exclusivamente a este propósito.

Las principales novedades del texto afectan a las normas comunes a las sociedades cooperativas en cuestiones como las relativas al capital social mínimo, el contenido mínimo de los Estatutos o la adaptación del silencio positivo a la Ley 39/2015. Junto a estas reformas es preciso destacar también las relativas a las clases de cooperativas y las formas de cooperación, como la supresión de los grupos de cooperativas, las cooperativas de trabajo o las cooperativas Agroalimentarias.

Palabras ClaVe: Economía Social, Sociedades cooperativas, Economía Social.

\begin{abstract}
The Law 4/2002, of April 11, of Cooperatives of the Community of Castilla y León reforms the Law 2/2018, of June 18, directed exclusively for this purpose.

The main novelties of the text affect the rules common to cooperative societies in matters such as those related to minimum social capital, the minimum content of the Statutes or the adaptation of positive silence to Law 39/2015. Along with these reforms, it is also necessary to highlight those related to cooperative classes and forms of cooperation, such as the suppression of cooperative groups, worker cooperatives or Agrofood cooperatives.
\end{abstract}

KEYwords: Social Economy, Cooperative societies, Social Economy. 
SUMARIO: 1. PRESENTACIÓN Y PLANTEAMIENTO. 2. CONTENIDO Y ALCANCE DE LA REFORMA. 2.1. Cambios en las normas comunes a las sociedades cooperativas. a) Capital social mínimo. b) Contenido mínimo de los Estatutos. c) Adaptación del silencio positivo a la Ley 39/2015. d) Respecto de los socios. e) Órganos de la cooperativa. f) Asamblea General. g) Régimen económico. h) Educación y Promoción. i) Reducción de cargas administrativas. 2.2. Modificaciones relativas a las clases de cooperativas y otras formas de cooperación. a) Supresión de los grupos de cooperativas. b) Cooperativas de trabajo. c) Cooperativas Agroalimentarias. d) Cooperativas de viviendas. e) Cooperativas de crédito y cooperativas de seguros. f) Un nuevo tipo de cooperativa: la "cooperativa integral". 3. VALORACIÓN DE CONJUNTO.

TABLE OF CONTENTS: 1. PRESENTATION AND APPROACH. 2. CONTENT AND SCOPE OF THE REFORM. 2.1. Changes in common standards to cooperative societies. a) Minimum social capital. b) Minimum content of the Statutes. c) Adaptation of positive silence to Law 39/2015. d) Regarding the partners. e) Organs of the cooperative. f) General Assembly. $g$ ) Economic regime. h) Education and Promotion. i) Reduction of administrative burdens. 2.2. Modifications related to cooperative classes and other forms of cooperation. a) Suppression of cooperative groups. b) Work cooperatives. c) Agri-Food Cooperatives. d) Housing cooperatives. e) Credit cooperatives and insurance cooperatives. f) A new type of cooperative: the "integral cooperative”. 3. OVERALL ASSESSMENT.

\section{Presentación y Planteamiento}

$\mathrm{E}$

1 BOE número 162, del pasado 5 de julio de 2018 hacía publica la reforma de la vigente Ley de cooperativas de Castilla y León, la Ley 4/2002, de 11 de abril, de Cooperativas de la Comunidad de Castilla y León, a través de la aprobación de la Ley $2 / 2018$, de 18 de junio, dirigida exclusivamente a este propósito ${ }^{1}$.

Esta reforma, que no es la primera de este cuerpo legal, ${ }^{2}$ se realiza, como se manifiesta en su propia Exposición de Motivos "en aras a simplificar y agilizar el funcionamiento de las cooperativas, teniendo en cuenta asimismo los requeri-

\footnotetext{
1 En 2019, las Cortes de Castilla y León tramitaros 9 leyes y 4 Decretos-leyes.

2 La Ley 4/2002, de 11 de abril, fue modificada por primera vez por la Ley 9/2004, de 28 de diciembre, de medidas económicas, fiscales y administrativas, que modificó los artículos 27, 103, 132, 141 y la disposición transitoria segunda, en lo concerniente al socio inactivo, a la prestación mínima de servicio del socio trabajador, al carácter constitutivo de la modificación de los Estatutos, a las uniones de cooperativas y a la ampliación del plazo de adaptación de los Estatutos. Posteriormente se modificó por la Ley 6/2011, de 4 de noviembre, para adaptarla a las nuevas normas del Sistema Contable Internacional. Por último, la Ley 5/2014, de 11 de septiembre, de medidas para la reforma de la Administración de la Comunidad de Castilla y León, dentro de sus «medidas organizativas» modificó los artículos 94, 134, 143 y 145 especialmente lo relativo a la racionalización de los órganos colegiados de asesoramiento y derogó los artículos 146 y 147, dejándolos sin contenido.
} 
mientos demandados por el propio sector." Se trata, sin embargo, de una reforma de cierta importancia, pues "afecta a cuarenta y cuatro artículos e incorpora dos disposiciones transitorias y dos finales. De los artículos modificados, la mayor parte, veintiocho, se refieren al título I «De la sociedad cooperativa. Normas Comunes» y otros doce al título II «De las clases de cooperativas y otras formas de cooperación»."

\section{CONTENIDO Y ALCANCE DE LA REFORMA}

Por lo que respecta a las modificaciones concretas incorporadas al texto legal, las mismas se pueden exponer del siguiente modo: 1) Cambios en el Título I (De la Sociedad Cooperativa. Normas Comunes) y 2) Modificaciones en el Título II (De las clases de cooperativas y otras formas de cooperación).

\subsection{Cambios en las normas comunes a las sociedades cooperativas}

\section{a) Capital social mínimo}

Uno de los principales cambios introducidos en esta reforma afecta a un dato esencial en cualquier sociedad mercantil, como es la fijación del capital social mínimo que se eleva de los 2.000 euros de la versión anterior y se sitúa a partir de ahora en 3.000 euros (Artículo 4), cuantía exigible anteriormente sólo para las cooperativas de viviendas. Como se precisa en la Exposición de Motivos (EM) del nuevo texto legal "Se equipara así al capital mínimo de las sociedades de capital y de las sociedades laborales.", lo cual no es sólo positivo en términos sistemáticos sino que se acerca a la realidad social de las diversas formas de sociedad mercantil, sin contar obviamente las sociedades anónimas y las sujetas a normativa especial.

\section{b) Contenido mínimo de los Estatutos}

En este punto, la reforma suprime la inclusión de la cláusula de sometimiento al arbitraje cooperativo (nueva redacción apartado 5, Artículo 6 Secciones), y lo hace, como también precisa la EM "debido a su escaso desarrollo práctico, sin perjuicio de que las Uniones, Federaciones y Confederación de Cooperativas puedan ejercer sus funciones de conciliación y arbitraje."

\section{c) Adaptación del silencio positivo a la Ley 39/2015}

La reforma se aprovecha también para atribuir valor positivo al silencio administrativo, en consonancia con lo establecido en el artículo 24 de la Ley 39/2015, de 1 de octubre, del Procedimiento Administrativo Común de las Administraciones Públicas en la calificación previa de los Estatutos (Artículo 15), donde se 
establece que de no recaer resolución expresa dentro del plazo de un mes, se entenderá el silencio como estimatorio. Idéntico plazo y, en la misma línea (silencio positivo) reformando el Artículo 17 respecto a la solicitud de inscripción registral de la cooperativa, teniendo en cuenta el valor constitutivo de ésta.

\section{d) Respecto de los socios}

La reforma introduce diversas modificaciones en lo que respecta al estatuto de los socios (derechos, deberes, clases...). Tal es el caso, de la baja voluntaria. Respecto de la que se incorpora la posibilidad de que, para determinados supuestos, pueda establecerse un período mayor para que el socio pueda darse de baja voluntaria (apartados 2 y 3 del Artículo 20).

En cuanto a los Derechos de los socios (Artículo 22), se reduce el plazo máximo previsto con anterioridad en la ley para proporcionar la información relativa a la situación económica de la cooperativa y los acuerdos del Consejo Rector, que pasa de tres meses a cuarenta y cinco días.

Asimismo se introduce una nueva clase de socios, al incluir al "socio de servicios." (Artículo 26), que se añade a la figura del "Socio colaborador", y al que se define (Artículo 26.2), como el "sin realizar la actividad principal, podrán participar de otras actividades o servicios que preste la cooperativa." previéndose que "[e]statutariamente se determinará el régimen de admisión y baja, así como los derechos y obligaciones de estos socios." (socio colaborador y socio de servicios) y se suprimen los límites establecidos en la redacción anterior de este artículo, remitiendo la regulación de los límites de votos y de las aportaciones al capital social a lo establecido en los artículos 35.6 y 59.5 de la ley.

\section{e) Órganos de la cooperativa}

Por lo que se refiere a los órganos de la cooperativa, la reforma introduce la posibilidad de utilización de medios electrónicos para determinados actos relativos al funcionamiento de la cooperativa, como la convocatoria de la Asamblea General regulada en el Artículo 32.2, o la publicación en la página web de la cooperativa, si la cooperativa tiene más de 500 socios.

\section{f) Asamblea General}

La nueva regulación da, como también precisa la EM de la nueva ley, una mayor concreción a las competencias de la Asamblea General recogidas en el Artículo 31.2, y a los aspectos relativos a la constitución y funcionamiento de la cooperativa regulados en los apartados 4 y 6 del Artículo 34, estableciéndose la posibilidad de votación secreta para el supuesto de que sea solicitado por al menos un $20 \%$ de los votos de los socios presentes y representados, unificándose 
con otros porcentajes establecidos en el mismo artículo, como el establecido en el apartado 7.

Asimismo, se regulan los límites de voto en el Artículo 35, incluyendo a los socios de servicios (que como señalamos, en una nueva clase de socio introducida por esta reforma) teniendo en cuenta la menor participación en la actividad cooperativizada.

Se modifica el Artículo 46 determinando que sean los Estatutos de la cooperativa los que establezcan el número de Interventores previendo la posibilidad de que en los mismos se disponga la no obligatoriedad de dicha figura en aquellas cooperativas sujetas a auditoría de cuentas.

Asimismo se introduce un nuevo apartado 4 al Artículo 53 extendiendo la figura de los delegados nombrados por la Asamblea General ordinaria cuando así lo prevean los Estatutos a las Asambleas extraordinarias que, en su caso, se celebren hasta la siguiente Asamblea ordinaria.

\section{g) Régimen económico}

En relación a este fundamental tema, la nueva regulación se acomoda el límite de aportaciones incorporando la nueva clase de socio de servicios y se condiciona el reembolso de éstas en el Artículo 66, para los supuestos en los que una cooperativa haya realizado cuantiosas inversiones, estableciéndose la posibilidad de un período mayor para que el socio pueda darse de baja sin causar un gran quebranto a ésta.

\section{h) Educación y Promoción}

Este aspecto del régimen singular de las cooperativas es también objeto de atención. Así, se suprime en el Artículo 72.3, relativo al Fondo de Educación y Promoción, las aportaciones que procedan del $20 \%$ de los resultados de operaciones con terceros. La justificación se recoge en la EM cuando afirma que estas aportaciones "no se contemplan en la normativa estatal, ni en la de comunidades autónomas, y pueden dar lugar a una mayor solvencia de la cooperativa."

También incide en el régimen económico de las cooperativas la modificación introducida en la aplicación de excedentes (apartado 1 del Artículo 74) que establece ahora la posibilidad de la reducción del Fondo de Reserva Obligatorio siempre y cuando la cuantía del mismo triplique al capital social.

\section{i) Reducción de cargas administrativas}

En esta materia, se introducen modificaciones en la legalización de los libros de las cooperativas y de las cuentas anuales, imponiéndose la obligatoriedad de su legalización de forma electrónica (Artículo 76.2 y 4). La presentación de las 
cuentas anuales se realizará también de forma electrónica, incluyendo la ampliación del tracto sucesivo respecto a éstas, de tal manera que transcurrido un año desde la fecha del cierre del ejercicio social sin que se hayan depositado desde la fecha del cierre del ejercicio social sin que se hayan depositado en el Registro de Cooperativas, éste no practicará inscripción alguna hasta que con carácter previo se practique su depósito, salvo las excepciones contempladas en el mismo. (Artículo 77.4 y 5 ).

\subsection{Modificaciones relativas a las clases de cooperativas y otras formas de cooperación}

\section{a) Supresión de los grupos de cooperativas}

La reforma suprimía los grupos de cooperativas (Artículo 98.2) que, paradójicamente, había sido una novedad destacada en la Ley $4 / 2002^{3}$ que clasificaba a las cooperativas en tres grupos, cooperativas de trabajadores, de servicios a los socios y de consumo. Su justificación, también expuesta en la EM de la Ley se encuentra en "que en la actualidad no se corresponden exactamente con las clases que agrupaban" y se incluye la clase de cooperativas integrales, al existir cooperativas que agrupan a varias clases de las ya existentes ${ }^{4}$.

\section{b) Cooperativas de trabajo}

En particular, y respecto a las cooperativas de trabajo, se acepta la posibilidad de cooperativas de trabajo con dos socios trabajadores (Artículo 100), "en consonancia con lo establecido en la normativa de otras comunidades autónomas, y con las peticiones realizadas por el propio sector. Se amplía el número máximo

3 En su EM se podía leer "La Ley de cooperativas de la Comunidad Autónoma de Castilla y León establece, desde el punto de vista de la actividad, como novedad, con ánimo clarificador una clasificación de las cooperativas en tres grupos, cooperativas de trabajadores, de servicios a los socios y de consumo."

4 De este modo, el nuevo Articulo 98.1 dispone: “ $« 1$. Las cooperativas de primer grado, de conformidad con el artículo 5 se clasifican en:

a) Cooperativas de trabajo.

b) Cooperativas de explotación comunitaria de la tierra y/o del ganado.

c) Cooperativas de enseñanza.

d) Cooperativas agroalimentarias.

e) Cooperativas de transportistas.

f) Cooperativas de industriales o de profesionales.

g) Cooperativas de viviendas.

h) Cooperativas de crédito.

i) Cooperativas de seguros.

j) Cooperativas de consumidores y usuarios.

k) Cooperativas integrales.»" 
de horas/año a realizar por los trabajadores por cuenta ajena al 50 por ciento". Se elimina, por tanto, la solicitud de superación del 30 por ciento anterior.

Asimismo, se establece que los Estatutos o el Reglamento de Régimen Interno aprobados en Asamblea General, regularán la actividad laboral, respetando la legislación laboral vigente y los convenios colectivos que resulten de aplicación y en las cooperativas con más de 25 socios trabajadores recogerán las condiciones de protección social de los socios trabajadores, para aquellas situaciones en las que el sistema de protección público no cubra total o parcialmente las necesidades de éstos (Artículo 103) 5 .

\section{c) Cooperativas Agroalimentarias}

Esta modificación "actualiza" la denominación de "Cooperativas Agrarias" en favor de la de "Cooperativas Agroalimentarias" (Artículo 113 y concordantes, pues se ha incorporado la misma al conjunto de la ley), probablemente como consecuencia de la integración vertical que progresivamente han experimentado, pasando de meros productores a transfortmadotres e incluso, comercializadoras. Así se expresa en la EM al afirmar que "El término agroalimentaria refleja mejor las actividades que pueden desarrollar esta clase de cooperativas, sean de carácter primario o bien de transformación de productos. Se amplían las actividades que pueden realizar como cooperativas y las relaciones entre socios y las cooperativas; aspectos clarificados con la nueva redacción del apartado 1 de dicho precepto."

\section{d) Cooperativas de viviendas}

Por lo que se refiere a las cooperativas de viviendas reguladas en el Artículo 118, al que se da una nueva redacción, estableciéndose un porcentaje mínimo de socios inscritos sobre los previstos totales para la toma de determinadas decisiones sobre aspectos importantes en la vida de éstas. Asimismo, se incluye la figura de los "gestores profesionales de cooperativas", regulando mínimamente, las incompatibilidades, su autorización por la Asamblea y su régimen de responsabilidad.

A este sentido, el nuevo Artículo 118 dispone:

“[...]

\footnotetext{
5 La EM aclara "En este último supuesto, con la autorregulación estatutaria, que de ningún modo trata de sustituir el sistema de protección pública, se pretende su complementariedad, sobre todo teniendo en cuenta que el sistema de protección social público, puede establecer diferencias tanto en lo referente a las contingencias protegidas como en lo que concierne a la intensidad y duración en el tiempo de éstas, dependiendo de la asimilación de los socios trabajadores a un régimen de Seguridad Social u otro."
} 
7. Las cooperativas de viviendas podrán contratar los servicios de un gestor profesional al objeto de efectuar los actos de gestión ordinaria que sean necesarios para el desarrollo de su objeto social, sin perjuicio de las facultades que les correspondan a los órganos de la sociedad.

Al gestor profesional se le aplicarán las siguientes reglas:

a) Los gestores, que podrán ser personas físicas o jurídicas, estarán sometidos a las normas de la presente ley sobre incompatibilidades, incapacidades y prohibiciones de los miembros del Consejo Rector y los interventores, no pudiendo además ni ser socios ni ocupar cargo alguno en los órganos de la sociedad. No obstante, se podrá tener la condición de gestor en diversas cooperativas, previa autorización de la Asamblea General de cada una de ellas.

b) La contratación del gestor, y las condiciones del contrato, en particular la remuneración, deberá ser autorizada por la Asamblea General.

c) Los poderes que eventualmente se otorguen al gestor serán siempre revocables. Cualquier cláusula contenida en dichos poderes contraria a esta disposición, se tendrá por no puesta.

d) El gestor asumirá frente a la Administración Pública, la cooperativa, los socios y terceros, la responsabilidad que la ley establece para los promotores inmobiliarios. La autorización, aprobación o ratificación de la actuación del gestor por cualquier órgano de la cooperativa no le exime de esta responsabilidad.

Se aplicarán las disposiciones de seguros.”

\section{e) Cooperativas de crédito y cooperativas de seguros}

La nueva redacción del Artículo 122, relativo a la "Normativa aplicable" a las "Cooperativas de crédito y cooperativas de seguros" establece con mayor claridad, como se expone en la EM "la existencia de dos clases de cooperativas, siéndoles de aplicación también las normas que, con carácter general, regulan la actividad de las entidades de crédito y de las entidades aseguradoras.", lo que, por otro lado es lógico pues se trata de normativa estatal. Tal afirmación, sin embargo, nunca es superflua.

\section{f) Un nuevo tipo de cooperativa: la "cooperativa integral"}

La última novedad de la reforma llevada a cabo en la normativa de cooperativas de Castilla y León supone el estableciendo un nuevo tipo de cooperativa, la cooperativa integral (añadiendo un nuevo artículo 123 bis), para regular lo que en 
la EM se califica como "una realidad existente". Así conforme al nuevo Artículo 123 bis "Se consideran cooperativas integrales aquellas que con independencia de su clase, su actividad cooperativizada es doble o plural, cumpliendo las finalidades propias de diferentes clases de cooperativas en una misma sociedad, según acuerdo de sus Estatutos y con observancia de lo regulado para cada una de dichas actividades. En dichos casos su objeto social será plural y se beneficiará del tratamiento legal que le corresponda por el cumplimiento de dichos fines".

\section{VALORACIÓN DE CONJUNTO}

Aunque siempre hemos sido particularmente críticos con la dispersión normativa y las sucesivas reformas a los que nos tiene acostumbrados nuestro Poder Legislativo (tanto las Cortes Generales como los Parlamentos Autonómicos), que en sede de cooperativas ha dado lugar a una pluralidad de leyes cuyas diferencias difícilmente justifican tal pluralidad, lo cierto es que, al menos desde la perspectiva de la técnica normativa, la Ley 2/2018, de 18 de junio, por la que se modifica la Ley 4/2002, de 11 de abril, de Cooperativas de la Comunidad de Castilla y León constituye, si no probablemente una excepción, sí un modelo, pues no da lugar a una nueva ley, sino que modifica la vigente en aquello que ve necesario, y lo justifica en una Exposición de Motivos, cuya lectura nos da la pista, como debe ser, de cuál es el alcance de la reforma que a través del nuevo texto legal se produce. 